\title{
TOLERANCES INDUCED BY IRREDUNDANT COVERINGS
}

\author{
JOUNI JÄRVINEN AND SÁNDOR RADELECZKI
}

\begin{abstract}
In this paper, we consider tolerances induced by irredundant coverings. Each tolerance $R$ on $U$ determines a quasiorder $\lesssim_{R}$ by setting $x \lesssim_{R} y$ if and only if $R(x) \subseteq R(y)$. We prove that for a tolerance $R$ induced by a covering $\mathcal{H}$ of $U$, the covering $\mathcal{H}$ is irredundant if and only if the quasiordered set $\left(U, \lesssim_{R}\right)$ is bounded by minimal elements and the tolerance $R$ coincides with the product $\gtrsim_{R} \circ \lesssim_{R}$. We also show that in such a case $\mathcal{H}=\left\{\uparrow m \mid m\right.$ is minimal in $\left.\left(U, \lesssim_{R}\right)\right\}$, and for each minimal $m$, we have $R(m)=\uparrow m$. Additionally, this irredundant covering $\mathcal{H}$ inducing $R$ consists of some blocks of the tolerance $R$. We give necessary and sufficient conditions under which $\mathcal{H}$ and the set of $R$-blocks coincide. These results are established by applying the notion of Helly numbers of quasiordered sets.
\end{abstract}

\section{INTRODUCTION AND MOTIVATION}

In this paper, we consider tolerances (reflexive and symmetric binary relations) and, in particular, tolerances induced by irredundant coverings. The term tolerance relation was introduced in the context of visual perception theory by E. C. Zeeman [17, motivated by the fact that indistinguishability of "points" in the visual world is limited by the discreteness of retinal receptors.

Let $R$ be a tolerance on $U$ and suppose that $R$ is interpreted as a similarity relation such that $x R y$ means that $x$ and $y$ are similar in terms of the knowledge we have about them. Of course, each object is similar to itself, and if $x$ is similar to $y$, it is natural to assume that $y$ is similar to $x$. Similarity relations are not necessarily transitive: we may have a finite sequence of objects $x_{1}, x_{2}, \ldots, x_{n}$ such that each two consecutive objects $x_{i}$ and $x_{i+1}$ are similar, but $x_{1}$ and $x_{n}$ are very different from each other.

Next, we recall some elementary notions and facts about rough set theory and formal concept analysis from [10] and [7, respectively. Let $R$ be a tolerance on $U$. The upper $R$-approximation of a set $X \subseteq U$ is

$$
X^{\mathbf{\Delta}}=\{x \in U \mid R(x) \cap X \neq \emptyset\}
$$

and the lower $R$-approximation of $X$ is

$$
X^{\mathbf{\nabla}}=\{x \in U \mid R(x) \subseteq X\} .
$$

Here $R(x)=\{y \in U \mid x R y\}$ denotes the $R$-neighbourhood of the element $x$. Then, $X^{\mathbf{\Delta}}$ can be viewed as the set of elements that possible are in $X$,

Acknowledgement: The research of the second author was carried out as part of the TAMOP-4.2.1.B-10/2/KONV-2010-0001 project supported by the European Union, cofinanced by the European Social Fund. 
because in $X$ is at least one element similar to them. The set $X^{\boldsymbol{\nabla}}$ can be considered as the set of elements certainly in $X$, because for these elements, all elements similar to them are in $X$.

Let us denote $\wp(U)^{\boldsymbol{\nabla}}=\left\{X^{\boldsymbol{\nabla}} \mid X \subseteq U\right\}$ and $\wp(U)^{\mathbf{\Lambda}}=\left\{X^{\mathbf{\Delta}} \mid X \subseteq U\right\}$. The complete lattices $\left(\wp(U)^{\mathbf{\Lambda}}, \subseteq\right)$ and $\left(\wp(U)^{\boldsymbol{\nabla}}, \subseteq\right)$ are ortholattices. In $\wp(U)^{\mathbf{\Lambda}}$, the orthocomplementation is ${ }^{\perp}: X^{\boldsymbol{\Lambda}} \mapsto X^{\mathbf{\Lambda} \boldsymbol{\Lambda}}$, and the map ${ }^{\top}: X^{\boldsymbol{\nabla}} \mapsto X^{\boldsymbol{\nabla}} \boldsymbol{v}^{\mathbf{v}}$ is the orthocomplementation operation of $\wp(U)^{\boldsymbol{\nabla}}$, where for any $A \subseteq U$, $A^{c}=U \backslash A$ is the set-theoretical complement of $A$.

A formal context $\mathbb{K}=(G, M, I)$ consists of two sets $G$ and $M$, and a relation $I$ from $G$ to $M$. The elements of $G$ are called the objects and the elements of $M$ are called attributes of the context $\mathbb{K}$. For $A \subseteq G$ and $B \subseteq M$, we define $A^{\prime}=\{m \in M \mid g I m$ for all $g \in A\}$ and $B^{\prime}=\{g \in G \mid$ $g I m$ for all $m \in B\}$. A formal concept of the context $(G, M, I)$ is a pair $(A, B)$ with $A \subseteq G, B \subseteq M, A^{\prime}=B$, and $B^{\prime}=A$. We call $A$ the extent and $B$ the intent of the concept $(A, B)$. The set of all concepts of the context $\mathbb{K}=(G, M, I)$ is denoted by $\mathfrak{B}(\mathbb{K})$. The set $\mathfrak{B}(\mathbb{K})$ is ordered by

$$
\left(A_{1}, B_{1}\right) \leq\left(A_{2}, B_{2}\right) \Longleftrightarrow A_{1} \subseteq A_{2} \Longleftrightarrow B_{1} \supseteq B_{2} .
$$

With respect to this order, $\mathfrak{B}(\mathbb{K})$ forms a complete lattice, called the concept lattice of the context $\mathbb{K}$. If $I \subseteq U \times U$ is an irreflexive and symmetric relation, then the concept lattice $\mathfrak{B}(\mathbb{K})$ corresponding to the context $\mathbb{K}=(U, U, I)$ is a complete ortholattice.

If $R$ is a tolerance relation on $U$, then its complement $R^{c}=U \times U \backslash R$ is irreflexive and symmetric. Thus, the concept lattice $\mathfrak{B}(\mathbb{K})$ of the context $\mathbb{K}=\left(U, U, R^{c}\right)$ is a complete ortholattice. We proved in [11] that

$$
\mathfrak{B}(\mathbb{K})=\left\{\left(A, A^{\top}\right) \mid A \in \wp(U)^{\mathbf{\nabla}}\right\},
$$

where ${ }^{\top}$ is the orthocomplement defined in $\wp(U)^{\boldsymbol{}}$. Additionally, for $\left(A, A^{\top}\right) \in \mathfrak{B}(\mathbb{K})$, its orthocomplement is $\left(A^{\top}, A\right)$. As noted in [11, for any tolerance $R$, the complete ortholattices $\wp(U)^{\boldsymbol{\nabla}}, \wp(U)^{\mathbf{\Lambda}}$, and $\mathfrak{B}(\mathbb{K})$ are isomorphic.

Let $\mathcal{L}=(L, \leq)$ be a lattice with a least element 0 . The lattice $\mathcal{L}$ is atomistic, if any element of $L$ is the join of atoms below it. We proved in [11] that for any tolerance $R$ on $U$, the following conditions are equivalent:

(C1) $R$ is induced by an irredundant covering;

(C2) $\wp(U)^{\boldsymbol{\nabla}}$ and $\wp(U)^{\mathbf{\Lambda}}$ are atomistic complete Boolean lattices;

(C3) $\mathfrak{B}(\mathbb{K})$ is an atomistic complete Boolean lattice.

This means that knowing when $R$ is induced by an irredundant covering is important, because this fully characterises the case when the lattices $\wp(U)$, $\wp(U)^{\mathbf{\Lambda}}$, and $\mathfrak{B}(\mathbb{K})$ are atomistic complete Boolean lattices. Note also that if $R$ is a tolerance on $U$ induced by an irredundant covering, then the set of atoms of $\wp(U) \mathbf{\Lambda}$ is $\{R(x) \mid R(x)$ is a block $\}$. Because the lattice-join in $\wp(U)^{\mathbf{\Lambda}}$ is the set-theoretical union, each element of $\wp(U)^{\mathbf{\Lambda}}$ can be given as a union of some $R$-neighbourhoods forming a block. Notice that the basic notions, such as coverings and blocks, related to tolerances are formally defined in Section 2 .

In this paper, we continue the study of tolerances induced by an irredundant covering. Our current study is done in the setting of quasiordered 
sets. Clearly, each tolerance $R$ on $U$ defines a quasiorder by $x \lesssim_{R} y$ if and only if $R(x) \subseteq R(y)$. We show that for a tolerance $R$ induced by a covering $\mathcal{H}$ of $U$, the covering $\mathcal{H}$ is irredundant if and only if the quasiordered set $\left(U, \lesssim_{R}\right)$ is bounded by minimal elements and the tolerance $R$ coincides with the product $\gtrsim_{R} \circ \lesssim_{R}$. We also characterise the case when this $\mathcal{H}$ and the set of $R$-blocks coincide.

This paper is structured as follows. Section 2 recalls shortly the basic facts about tolerances. In Section 3, we consider the relationship between tolerances and quasiorders, and we characterise the case in which a tolerance is induced by an irredundant covering in terms of minimal elements of a quasiordered set. We adopt from combinatorics the notion of Helly numbers in Section 4, and we connect Helly numbers of quasiordered sets and blocks of a tolerance forming an irredundant covering. In particular, by using this result for a tolerance $R$ induced by an irredundant covering $\mathcal{H}$, we give necessary and sufficient conditions for $\mathcal{H}=\mathcal{B}(R)$, that is, for normality of the covering $\mathcal{H}$. The section ends with a consideration of tolerances induced by finite distributive lattices.

\section{Preliminaries}

Let us first recall some notions and basic results from [2,15]. Let $R$ be a tolerance on $U$, that is, $R$ is a reflexive and symmetric binary relation on $U$. A nonempty subset $X$ of $U$ is an $R$-preblock if $X^{2} \subseteq R$. An $R$-block is a maximal $R$-preblock, that is, an $R$-preblock $B$ is an $R$-block if $B \subseteq X$ implies $B=X$ for any $R$-preblock $X$. Thus, any subset $\emptyset \neq X \subseteq U$ is an $R$-preblock if and only if it is contained in some $R$-block. The family of all $R$-blocks is denoted by $\mathcal{B}(R)$. If there is no danger of confusion, we simply call $R$-blocks as blocks. Each tolerance $R$ is completely determined by its blocks, that is, $a R b$ if and only if there exists a block $B \in \mathcal{B}(R)$ such that $a, b \in B$. This can be written in the form $R=\bigcup\left\{B^{2} \mid B \in \mathcal{B}(R)\right\}$. It is also known that $B$ is a block if and only if

$$
B=\{x \in U \mid B \subseteq R(x)\} .
$$

We recall from [3, 14] the following lemma combining some conditions for an $R$-neighbourhood forming a block.

Lemma 2.1. Let $R$ be a tolerance on $U$ and $x \in U$. Then, the following are equivalent:

(a) $R(x)$ is a block;

(b) $a R b$ for all $a, b \in R(x)$;

(c) $a \in R(x)$ implies $R(x) \subseteq R(a)$;

(d) $R(x)=\bigcap\{R(a) \mid a \in R(x)\}$.

A collection $\mathcal{H}$ of nonempty subsets of $U$ is called a covering of $U$ if $\cup \mathcal{H}=U$. A covering $\mathcal{H}$ is irredundant if $\mathcal{H} \backslash\{X\}$ is not a covering for any $X \in \mathcal{H}$. Clearly, the blocks $\mathcal{B}(R)$ of a tolerance $R$ form a covering, but this covering is not generally irredundant. On the other hand, each covering $\mathcal{H}$ of $U$ defines a tolerance $R_{\mathcal{H}}=\bigcup\left\{X^{2} \mid X \in \mathcal{H}\right\}$, called the tolerance induced by $\mathcal{H}$. Obviously, the sets in $\mathcal{H}$ are preblocks of $R_{\mathcal{H}}$.

Let $R$ be a tolerance induced by a covering $\mathcal{H}$ of $U$. In [11, Proposition 3.7], we showed the equivalence of the following statements: 
(D1) $\mathcal{H}$ an irredundant covering of $U$;

(D2) $\mathcal{H} \subseteq\{R(x) \mid x \in U\}$.

Example 2.2. Let us consider the following setting modified from an example appearing in $[15$. Let $\mathbb{N}=\{1,2,3, \ldots\}$. We define a tolerance $R$ on $U=\wp(\mathbb{N}) \backslash\{\emptyset\}$ by setting for any nonempty subsets $B, C \subseteq \mathbb{N}$ :

$$
(B, C) \in R \Longleftrightarrow B \cap C \neq \emptyset .
$$

Let $n \in \mathbb{N}$ and define $K_{n}=\{B \in U \mid n \in B\}$. Clearly, $R(\{n\})=K_{n}$. Each $R(\{n\})=K_{n}$ is also a tolerance block, because all sets in $K_{n}$ contain $n$, and hence they are $R$-related (cf. Lemma 2.1).

Let $\mathcal{H}=\left\{K_{n} \mid n \in \mathbb{N}\right\}$. Then, $\bigcup \mathcal{H}=\wp(\mathbb{N}) \backslash\{\emptyset\}$, that is, $\mathcal{H}$ is a covering of $U$. The tolerance $R_{\mathcal{H}}$ induced by $\mathcal{H}$ is $R$, because if $(B, C) \in R$, then there exists $n \in B \cap C$ and so $B, C \in K_{n}$. Conversely, if $B, C \in K_{n}$, then $(B, C) \in R$, because $K_{n}$ is a block. Since each set in $\mathcal{H}$ is of the from $K_{n}=R(\{n\}), n \in \mathbb{N}$, the covering $\mathcal{H}$ is irredundant by the equivalence of (D1) and (D2).

Lemma 2.3. If $R$ is a tolerance induced by an irredundant covering $\mathcal{H}$, then $\mathcal{H} \subseteq \mathcal{B}(R)$.

Proof. Because conditions (D1) and (D2) are equivalent, there exists $Y \subseteq U$ such that $\mathcal{H}=\{R(y) \mid y \in Y\}$. Since $R$ is induced by $\mathcal{H}$, then for each $y \in Y$, we have $a R b$ for any $a, b \in R(y)$. Thus, by Lemma 2.1, $R(y)$ is a block for any $y$ in $Y$, and $\mathcal{H} \subseteq \mathcal{B}(R)$.

Let $R$ be a tolerance induced by an irredundant covering $\mathcal{H}$. The inclusion $\mathcal{H} \subseteq \mathcal{B}(R)$ presented in Lemma 2.3 can be proper (see Example 4.5). In Section 4, we give a characterisation for the case $\mathcal{H}=\mathcal{B}(R)$.

Example 2.4. Tolerances can be considered as simple graphs (and vice versa). A simple graph is an undirected graph that has no loops (edges connected at both ends to the same vertex) and no multiple edges. Any tolerance $R$ on $U$ determines a graph $\mathcal{G}=(U, R)$, where $U$ is interpreted as the set of vertices and $R$ as the set of edges. There is a line connecting $x$ and $y$ if and only if $x R y$. Because each point is $R$-related to itself, loops connecting a point to itself are not drawn/permitted. Note that [18] contains some elementary connections between tolerances and undirected graphs.

A nonempty set $X \subseteq U$ is a preblock if and only if $X$ is a clique of $\mathcal{G}$, that is, all points in $X$ are connected by an edge of $\mathcal{G}$. A block of $R$ is thus a maximal clique. For any $x \in U$, the neighbourhood $R(x)$ is the set of points connected to $x$. If this set forms a clique, then by Lemma 2.1, $R(x)$ is necessarily a block, that is, a maximal clique. In general, listing all maximal cliques may require exponential time as there exist graphs with exponentially many maximal cliques. The article [1] presents an improved version of the fundamental Harary-Ross algorithm [9] enumerating all cliques of a graph.

Note also that blocks (or maximal cliques) have several applications. For instance, in [12] are studied tolerances on lattices and their block description, and in [13] blocks of compatible relations on error algebras are considered. 


\section{TOleRANCES INDUCED BY QUASIORDERS}

Let $\lesssim$ be a quasiorder on $U$, that is, $\lesssim$ is a reflexive and transitive binary relation on $U$. The pair $(U, \lesssim)$ is called a quasiordered set. An element $x$ is a minimal element of $(U, \lesssim)$, if $y \lesssim x$ implies $y \gtrsim x$, where $\gtrsim$ denotes the inverse relation of $\lesssim$. Note that this definition coincides with the definition of minimality in partially ordered sets, because of the antisymmetry of a partial order $\leq$. We say that $(U, \lesssim)$ is bounded by minimal elements if for any $x \in U$, there exists a minimal $m \in U$ such that $m \lesssim x$.

The relation $\gtrsim 0 \lesssim$ is a tolerance on $U$ and it is denoted simply by $\approx$. The upset $\lesssim(x)=\{y \mid x \lesssim y\}$ is written as $\uparrow x$. Note that for any $x \in U, \uparrow x$ is a preblock of $\approx$, because $a, b \in \uparrow x$ is equivalent to $a \gtrsim x \lesssim b$, that is, $a \approx b$.

Lemma 3.1. Let $\lesssim$ be a quasiorder on $U$.

(a) If $m$ is a minimal element, then $\approx(m)=\uparrow m$ and $\uparrow m$ is a block of $\approx$.

(b) If $(U, \lesssim)$ is bounded by minimal elements, then $\mathcal{H}=\{\uparrow m \mid$ $m$ is minimal $\}$ is an irredundant covering of $U$, and the tolerance induced by $\mathcal{H}$ is $\approx$.

Proof. (a) We first show that $m \approx x$ if and only if $x \in \uparrow m$. If $x \approx m$, then $x \gtrsim a \lesssim m$ for some $a \in U$. But since $m$ is minimal, $a \lesssim m$ gives $m \lesssim a \lesssim x$ and $x \in \uparrow m$. Conversely, $x, m \in \uparrow m$ implies $x \approx m$ since $\uparrow m$ is a preblock of $\approx$. Thus, $\approx(m)=\uparrow m$.

If $x, y \in \uparrow m$, then $m \lesssim x$ and $m \lesssim y$ give $x \approx y$. By Lemma 2.1 this means that $\uparrow m$ is a block of $\approx$.

(b) Let $x \in U$. Because $U$ is bounded by minimal elements, there exists a minimal $m$ such that $m \lesssim x$ and $x \in \uparrow m$. Hence, $\bigcup \mathcal{H}=U$.

Next, we prove that the tolerance $R_{\mathcal{H}}$ induced by $\mathcal{H}$ equals $\approx$. If $(x, y) \in$ $R_{\mathcal{H}}$, then there exists a minimal $m$ such that $x, y \in \uparrow m$. Then, $x \gtrsim m \lesssim y$ gives $x \approx y$. Conversely, $x \approx y$ means that there exists an element $a$ such that $x \gtrsim a \lesssim y$. Thus, $a \lesssim x, y$. Since $U$ is bounded by minimal elements, there exists a minimal $m$ such that $m \lesssim a \lesssim x, y$. Therefore, $x, y \in \uparrow m$ and $(x, y) \in R_{\mathcal{H}}$.

Because each element of $\mathcal{H}$ is of the form $R_{\mathcal{H}}(m)=\approx(m)=\uparrow m, \mathcal{H}$ is irredundant, since (D1) and (D2) are equivalent.

Corollary 3.2. Let $(U, \lesssim)$ be bounded by minimal elements and $x, y \in U$. Then, $x \approx y$ if and only if there exists a minimal $m$ such that $x, y \in \uparrow m$.

Let $R$ be a tolerance on $U$. It is clear that the relation $\lesssim_{R}$ defined by

$$
x \lesssim_{R} y \Longleftrightarrow R(x) \subseteq R(y)
$$

is a quasiorder on $U$. In addition, we denote by $\approx_{R}$ the relation $\gtrsim_{R} \circ \lesssim_{R}$. It is easy to observe that

$$
x \approx_{R} y \Longleftrightarrow(\exists a) R(a) \subseteq R(x) \cap R(y) .
$$

Lemma 3.3. If $R$ is a tolerance on $U$ induced by an irredundant covering $\mathcal{H}$, then $\left(U, \lesssim_{R}\right)$ is bounded by the minimal elements $\{d \in U \mid R(d)$ is a block\}.

Proof. Let $R(d)$ be a block. As noted in Section 1, $R(d)$ is an atom of $\wp(U)$. Suppose that $x \lesssim_{R} d$. Then, $\emptyset \neq R(x) \subseteq R(d)$, and because $R(x) \in \wp(U)^{\boldsymbol{\Lambda}}$ and $R(d)$ is an atom of $\wp(U)^{\mathbf{\Lambda}}$, we have $R(d)=R(x)$. This gives $x \gtrsim_{R} d$, 
and thus $d$ is minimal. On the other hand, if $m$ is a minimal element, then for all $x \in U, x \lesssim_{R} m$ implies $x \gtrsim_{R} m$, that is, $R(x) \subseteq R(m)$ implies $R(x)=R(m)$. Because $R(m) \in \wp(U)^{\mathbf{\Lambda}}$, there exists an atom $R(d)$ of $\wp(U)^{\mathbf{\Lambda}}$ such that $R(d) \subseteq R(m)$. By the minimality of $m$, we have $R(m)=R(d)$. Since $R(d)$ is a block, also $R(m)$ is a block. Therefore, the set of minimal elements of $\left(U, \lesssim_{R}\right)$ is $\{d \in U \mid R(d)$ is a block $\}$.

Let $x \in U$. Since $R(x) \in \wp(U) \mathbf{\Lambda}$, there exists $R(d)$ such that $R(d)$ is a block and $R(d) \subseteq R(x)$. But $R(d) \subseteq R(x)$ means $d \lesssim_{R} x$ and since $d$ is minimal by the above, $U$ is bounded by the minimal elements.

Theorem 3.4. For a tolerance $R$ on $U$, the following are equivalent:

(a) $R$ is induced by an irredundant covering.

(b) $\left(U, \lesssim_{R}\right)$ is bounded by minimal elements and $R$ equals $\approx_{R}$.

Proof. (a) $\Rightarrow$ (b): By Lemma 3.3. $\left(U, \lesssim_{R}\right)$ is bounded by minimal elements. Thus, it suffices to show that $R$ equals $\approx_{R}$. If $a R b$, then by Lemma 2.3 and the equivalence of (D1) and (D2), there exists $d$ such that $R(d)$ is a block of $R$ and $a, b \in R(d)$. By Lemma 2.1, we have $R(d) \subseteq R(a) \cap R(b)$, and hence $a \approx_{R} b$. Conversely, if $a \approx_{R} b$, then by Corollary 3.2, there exists a $\lesssim R$-minimal element $d$ such that $a, b \in \uparrow d$, that is, $R(d) \subseteq R(a), R(b)$. Notice that by Lemma 3.3, $d$ is an element such that $R(d)$ is a block. Then, $R(d) \subseteq R(a), R(b)$ implies $a, b \in R(d)$, and since $R(d)$ is a block, we have $a R b$.

(b) $\Rightarrow(\mathrm{a})$ : If $\left(U, \lesssim_{R}\right)$ is bounded by minimal elements, then by Lemma 3.1 (b), the tolerance $R=\approx_{R}$ is induced by the irredundant covering $\mathcal{H}=\{\uparrow m \mid m$ is minimal $\}$.

Example 3.5. For a given tolerance $R$ on $U$, the minimal elements $x$ in $\left(U, \lesssim_{R}\right)$ are such that $R(x)$ is a block, as is shown in the proof of Lemma 3.3 , In Example 2.4, we noted that $R(x)$ is a block if and only if $R(x)$ is a clique.

Consider the graph $\mathcal{G}$ defined by the tolerance $R$ on $U=\{a, b, c, d\}$ depicted in Figure 1, Note that this graph originates from Example 1.3 in [14]. The $R$-neighbourhood of the elements $a, b$, and $d$ forms a clique. Thus, they are minimal in $\left(U, \lesssim_{R}\right)$. Note that the elements $a$ and $b$ are such that $a \lesssim_{R} b$

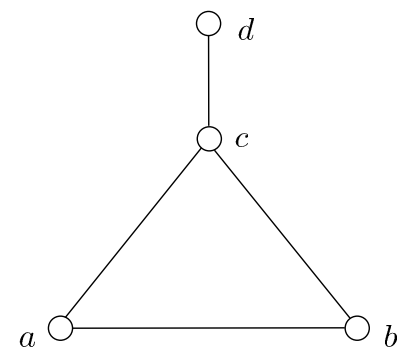

$\mathcal{G}=(U, R)$

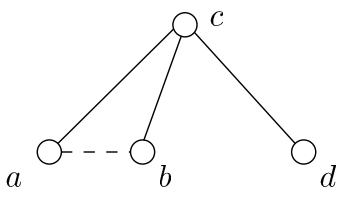

$\left(U, \lesssim_{R}\right)$

Figure 1.

and $b \lesssim_{R} a$, but $a \neq b$. This is expressed in the diagram of $\left(U, \lesssim_{R}\right)$ by the dashed segment, and the solid lines depict the "natural" partial order between the elements. Because $U$ is bounded by minimal elements and $\approx_{R}$ 
is equal to $R$, the family $\{\{a, b, c\},\{c, d\}\}$ of the $R$-neighbourhoods of the minimal elements form an irredundant covering inducing $R$ by Theorem 3.4 .

Remark 3.6. Let $R$ be a tolerance induced by an irredundant covering $\mathcal{H}$. Since the sets in $\mathcal{H}$ are of the form $\uparrow m=R(m)$, where $m$ is a minimal element of $\left(U, \lesssim_{R}\right)$, we have that $x R y$ if and only if there exists a minimal element element $m$ in $\left(U, \lesssim_{R}\right)$ such that $x R m$ and $y R m$. Thus, the minimal elements can be considered as a "model" of the tolerance $R$, because $R$ is completely determined by the $R$-neighbourhoods of the minimal elements. In [11], we called these elements complete. We also know that $\mathcal{H} \subseteq \mathcal{B}(R)$ and this inclusion can be proper. Thus, there can be such blocks of $R$ that are not determined by a complete element. In Section 4 , we will concentrate on characterising the case in which $\mathcal{H}=\mathcal{B}(R)$.

We end this section considering shortly the notion of a canonical base introduced in [15]. Let $R$ be a tolerance on $U$. A subset $\mathcal{K} \subseteq \mathcal{B}(R)$ is called a canonical base of $R$ if $\mathcal{K}$ induces $R$, but for any $X \in \mathcal{K}$, the family $\mathcal{K} \backslash\{X\}$ does not induce $R$.

If $R$ is induced by an irredundant covering of $\mathcal{H}$, then $\mathcal{H} \subseteq \mathcal{B}(R)$, as we noted already. In addition, $\mathcal{H}$ consists of the upsets $\uparrow m$ of the minimal elements of $\left(U, \lesssim_{R}\right)$. This implies that $\mathcal{H}$ is a canonical base, because if we remove $\uparrow m$ from $\mathcal{H}$, then the pair $(m, m)$ does not belong to the relation induced by the family $\mathcal{H} \backslash\{\uparrow m\}$, but trivially $(m, m) \in R$.

Proposition 3.7. Let $R$ be a tolerance induced by a family $\mathcal{H} \subseteq \mathcal{B}(R)$. Then, the following assertions are equivalent:

(a) $\mathcal{H}$ is an irredundant covering of $U$;

(b) $\mathcal{H}$ is the unique canonical base and $\mathcal{H} \subseteq\{R(x) \mid x \in U\}$.

Proof. (a) $\Rightarrow(\mathrm{b})$ : If $\mathcal{H}$ is an irredundant covering of $U$, then $\mathcal{H} \subseteq\{R(x) \mid x \in$ $U\}$ and $\mathcal{H}$ is a canonical base. Let $\mathcal{K} \subseteq \mathcal{B}(R)$ be an arbitrary canonical base of $R$. Then, $\mathcal{K}$ is also a covering of $U$, and hence for any minimal element $m$ of $\left(U, \lesssim_{R}\right)$, there exists a block $B \in \mathcal{K}$ such that $m \in B$. Because $B$ is a block, $B \subseteq R(m) \in \mathcal{H}$. Since $R(m)$ is also a block of $R$, we get $R(m)=B$. Thus $\mathcal{H} \subseteq \mathcal{K}$, and we get $\mathcal{H}=\mathcal{K}$, because $\mathcal{K}$ is a canonical base.

(b) $\Rightarrow$ (a): The reflexivity of $R$ implies that any canonical base is a covering of $U$. Because the covering $\mathcal{H}$ satisfies condition (D2), it is irredundant.

Example 3.8. In Proposition 3.7, we showed that if $R$ is a tolerance induced by an irredundant covering, then this covering is also the unique canonical base. In this example, we show that unique bases do not necessarily form irredundant coverings. Consider the tolerance $R$ on $\{a, b, c, d\}$ such that $R(a)=\{a, b\}, R(b)=\{a, b, c\}, R(c)=\{b, c, d\}$, and $R(d)=\{c, d\}$. The family $\mathcal{B}(R)=\{\{a, b\},\{b, c\},\{c, d\}\}$ of the blocks of $R$ is the unique canonical base of $R$, but $\mathcal{B}(R)$ does not form an irredundant covering of $U$, because the block $\{b, c\}$ can be removed and we still have a covering of $U$.

\section{Helly number AND IRREDUNDANT COVERINGS}

The Helly number of a family of sets $\mathcal{H}$ is $k$, if for any subfamily $\mathcal{S}$ of $\mathcal{H}$, any $k$ members of $\mathcal{S}$ have a common point, then the sets in $\mathcal{S}$ have a common point. This number is named after Edward Helly (1884-1943). Let us now 
define a similar notion in quasiordered sets. Let $(U, \lesssim)$ be a quasiordered set. We say that $(U, \lesssim)$ has the Helly number $k$, if for any subset $A \subseteq U$, any $k$ elements of $A$ have a common lower bound, then $A$ has a common lower bound. In particular, the Helly number of $(U, \lesssim)$ is 2 if and only if every preblock of $\approx$ has a lower bound.

Theorem 4.1. Let $(U, \lesssim)$ be a quasiordered set. Then, the following assertions are equivalent:

(a) The Helly number of $(U, \lesssim)$ is 2;

(b) $\mathcal{B}(\approx)=\{\uparrow m \mid m$ is minimal $\}$;

(c) $\mathcal{B}(\approx)$ is an irredundant covering and $(U, \lesssim)$ is bounded by minimal elements.

Proof. $(\mathrm{a}) \Rightarrow(\mathrm{b})$ : Let $B$ be a block of $\approx$. Because $U$ has the Helly number $2, B$ has a lower bound $m$. Thus, $B \subseteq \uparrow m$, and because $B$ is a block and $\uparrow m$ is a preblock, we have $B=\uparrow m$. This element $m$ is minimal, because if $a \lesssim m$, then $\uparrow m \subseteq \uparrow a$. Since $\uparrow m$ is a block and $\uparrow a$ is a preblock, we have $\uparrow m=\uparrow a$. Thus, $a \in \uparrow m$ gives $m \lesssim a$ and $m$ is minimal. On the other hand, if $m$ is minimal, then $\uparrow m$ is a block by Lemma 3.1(a). Thus, $\mathcal{B}(\approx)=\{\uparrow m \mid m$ is a minimal $\}$.

(b) $\Rightarrow$ (c): If $\mathcal{B}(\approx)=\{\uparrow m \mid m$ is minimal $\}$, then for all $x \in U$, there exists a minimal $m_{x}$ such that $x \in \uparrow m_{x}$ and $m_{x} \lesssim x$. Thus, $(U, \lesssim)$ is bounded by minimal elements. By Lemma 3.1(b), the covering $\mathcal{B}(\approx)$ is irredundant.

$(\mathrm{c}) \Rightarrow(\mathrm{a})$ : Assume that $\mathcal{B}(\approx)$ is an irredundant covering and $(U, \lesssim)$ is bounded by minimal elements. Then, by Lemma 3.1(b), $\mathcal{H}=\{\uparrow m \mid$ $m$ is minimal $\}$ is an irredundant covering inducing $\approx$. Lemma 2.3 implies $\mathcal{H} \subseteq \mathcal{B}(\approx)$. But since also $\mathcal{B}(\approx)$ is irredundant, we must have $\mathcal{H}=\mathcal{B}(\approx)$. Suppose $P$ is a $\approx$-preblock. Then, there exists a block $B$ of $\approx$ such that $P \subseteq B \in \mathcal{H}$. Because $\mathcal{H}$ consists of such upsets $\uparrow m$ that $m$ is minimal, there exists a minimal $m$ such that $B=\uparrow m$. Thus, $m$ is a lower bound of $B$ and $P$, and the Helly number of $(U, \lesssim)$ is 2 .

In the following, we characterise for finite quasiordered sets the cases in which their Helly number is 2 . If $(U, \lesssim)$ is a finite quasiordered set, then $(U, \lesssim)$ has at least one minimal element. This can be seen as follows. Let $a_{1}$ be any element of $U$. If $a_{1}$ is not minimal, then there exists an element $a_{2}$ such that $a_{2} \lesssim a_{1}$, but $a_{2} \gtrsim a_{1}$. If $a_{2}$ is minimal, then we are finished. If $a_{2}$ is not minimal, then there is $a_{3}$ such that $a_{3} \lesssim a_{2}$ and $a_{3} \gtrsim a_{2}$. If $a_{3}$ is not minimal, continue this procedure. Since $A$ is finite, this process must terminate.

Proposition 4.2. Let $(U, \lesssim)$ be a finite quasiordered set. The following are equivalent:

(a) The Helly number of $(U, \lesssim)$ is 2 .

(b) If for some minimal elements $a_{1}, a_{2}, a_{3} \in U$, the intersections $\uparrow a_{1} \cap \uparrow a_{2}$, $\uparrow a_{1} \cap \uparrow a_{3}, \uparrow a_{2} \cap \uparrow a_{3}$ are nonempty, then there exists a minimal element $a \in U$ such that

$$
\left(\uparrow a_{1} \cap \uparrow a_{2}\right) \cup\left(\uparrow a_{1} \cap \uparrow a_{3}\right) \cup\left(\uparrow a_{2} \cap \uparrow a_{3}\right) \subseteq \uparrow a .
$$

Proof. (a) $\Rightarrow$ (b): Let $a_{1}, a_{2}, a_{3}$ be minimal elements and let $H=\left(\uparrow a_{1} \cap\right.$ $\left.\uparrow a_{2}\right) \cup\left(\uparrow a_{1} \cap \uparrow a_{3}\right) \cup\left(\uparrow a_{2} \cap \uparrow a_{3}\right)$. If $x, y \in H$, then there exists $a_{i}$ for some 
$1 \leq i \leq 3$ such that $x \gtrsim a_{i} \lesssim y$ and $x \approx y$. This means that $H$ is a preblock, and since (a) holds, $H$ has a lower bound $b$. Because $(U, \lesssim)$ is finite, there exists a minimal element $a$ such that $a \lesssim b$. Hence, $H \subseteq \uparrow a$.

(b) $\Rightarrow($ a): We show by induction that each preblock $X$ of $\approx$ has a lower bound. Let $X$ be a preblock. If $|X|=1$, then the only element in $X$ is trivially a lower bound of $X$. If $|X|=2$, then $X=\{x, y\}$ for some $x, y \in U$, and $x \approx y$ means that there is $a \in U$ such that $a \lesssim x, y$. Thus, $a$ is a lower bound for $X$. As an induction hypothesis, we assume that each preblock $X$ of $\approx$ such that $|X| \leq n$ has a lower bound. Let us consider a preblock $X$ such that $|X|=n+1$. Since $X$ has now at least three elements, $X$ can be partitioned into three disjoint nonempty sets $X_{1}, X_{2}, X_{3}$. It is obvious that the sets $X_{1} \cup X_{2}, X_{1} \cup X_{3}, X_{2} \cup X_{3}$ are preblocks having at most $n$ elements. By the induction hypothesis, these preblocks have lower bounds. Since $(U, \lesssim)$ is finite, there exists minimal elements $a_{1}, a_{2}, a_{3} \in U$ such that $a_{1} \lesssim x_{1}$ for all $x_{1} \in X_{1} \cup X_{2}, a_{2} \lesssim x_{2}$ for all $x_{2} \in X_{1} \cup X_{3}$, and $a_{3} \lesssim x_{3}$ for all $x_{3} \in X_{2} \cup X_{3}$. This implies that

$$
X_{1} \subseteq\left(\uparrow a_{1} \cap \uparrow a_{2}\right), X_{2} \subseteq\left(\uparrow a_{1} \cap \uparrow a_{3}\right), X_{3} \subseteq\left(\uparrow a_{2} \cap \uparrow a_{3}\right)
$$

Because the intersections $\uparrow a_{1} \cap \uparrow a_{2}, \uparrow a_{1} \cap \uparrow a_{3}, \uparrow a_{2} \cap \uparrow a_{3}$ are nonempty and (b) holds, there exists a minimal element $a$ such that

$$
X=X_{1} \cup X_{2} \cup X_{3} \subseteq\left(\uparrow a_{1} \cap \uparrow a_{2}\right) \cup\left(\uparrow a_{1} \cap \uparrow a_{3}\right) \cup\left(\uparrow a_{2} \cap \uparrow a_{3}\right) \subseteq \uparrow a .
$$

Thus, the preblock $X$ has a lower bound $a$.

We will call a covering $\mathcal{H}$ of a set $U$ normal if it satisfies the conditions (ii) and (iv) from page 19 of the book [3], that is,

(N1) $\mathcal{H}$ is an antichain with respect to $\subseteq$.

(N2) If $M \subseteq U$ is a set such that $M \nsubseteq B$ for all $B \in \mathcal{H}$, then there exists at least one two-element set $\{a, b\} \subseteq M$ such that $\{a, b\} \nsubseteq B$ for all $B \in \mathcal{H}$.

Theorem 2.9 of [3] (see also [4-6, 8]) states that a covering $\mathcal{H}$ of $U$ is normal if and only if there is a tolerance $R$ on $U$ such that $\mathcal{H}=\mathcal{B}(R)$. For a normal covering $\mathcal{H}$, this $R$ is just the tolerance induced by $\mathcal{H}$. Thus, there is a one-to-one correspondence between tolerances on $U$ and normal coverings on $U$. Note also that in [19, normal coverings, and their relationship to cliques of a graph, are studied under the name $\tau$-coverings. As an immediate consequence of [3] and [19], we obtain:

Remark 4.3. Let $\mathcal{H}$ be a normal covering of $U$. If for some $B_{1}, B_{2}, B_{3} \in \mathcal{H}$, the intersections $B_{1} \cap B_{2}, B_{1} \cap B_{3}$, and $B_{2} \cap B_{3}$ are nonempty, then there exists $B \in \mathcal{H}$ that includes these intersections. Indeed, let $C=\left(B_{1} \cap B_{2}\right) \cup$ $\left(B_{1} \cap B_{3}\right) \cup\left(B_{2} \cap B_{3}\right)$. Then, for any $\{a, b\} \subseteq C$, we have $\{a, b\} \subseteq B_{i}$ for some $i \in\{1,2,3\}$. Since $\mathcal{H}$ is a normal covering of $U, C \nsubseteq B$ for all $B \in \mathcal{H}$ is not possible. Therefore, there exists $B \in \mathcal{H}$ that includes $C$.

In Lemma 2.3, we showed that if $R$ is a tolerance induced by an irredundant covering $\mathcal{H}$, then $\mathcal{H} \subseteq \mathcal{B}(R)$. The natural question then is, when such a $\mathcal{H}$ coincides with $\mathcal{B}(R)$ ? An obvious answer is that exactly in the case the covering $\mathcal{H}$ is normal. Next we present a theorem that connects normal coverings, Helly numbers of 2, and the condition given in Remark 4.3 for 
tolerances induced by an irredundant finite covering. For that, we need to introduce some notation. Let $R$ be a tolerance on $U$. We denote by

$$
\mathcal{N}=\{R(x) \mid x \in U\}
$$

the set of all $R$-neighbourhoods of the elements of $U$. This set can be ordered by the set-inclusion $\subseteq$. Thus, $(\mathcal{N}, \subseteq)$ is a partially ordered set. Observe that $x \lesssim_{R} y$ in $U$ if and only if $R(x) \subseteq R(y)$ in $\mathcal{N}$. The following conditions are now obvious:

- The element $m \in U$ is a minimal element of $\left(U, \lesssim_{R}\right)$ if and only if $R(m)$ is a minimal element of $(\mathcal{N}, \subseteq)$.

- The Helly number of $\left(U, \lesssim_{R}\right)$ is 2 if and only if the Helly number of $(\mathcal{N}, \subseteq)$ is 2 .

If $R$ is a tolerance induced by a finite irredundant covering $\mathcal{H}$ of $U$, then $\mathcal{N}$ is finite. Indeed,

$$
\mathcal{H}=\{R(m) \mid m \text { is minimal }\}=\left\{R(m) \mid R(m) \text { is an atom of } \wp(U)^{\mathbf{\Lambda}}\right\}
$$

and hence $\wp(U) \boldsymbol{\Lambda}$ is finite, because it is generated by a finite set $\mathcal{H}$ of atoms. Therefore, $\mathcal{N} \subseteq \wp(U)^{\mathbf{\Lambda}}$ must be finite.

Proposition 4.4. Let $R$ be a tolerance induced by a finite irredundant covering $\mathcal{H}$ of $U$. Then, the following assertions are equivalent:

(i) $\mathcal{H}=\mathcal{B}(R)$.

(ii) $\mathcal{H}$ is a normal covering.

(iii) If for some $B_{1}, B_{2}, B_{3} \in \mathcal{H}$, the intersections $B_{1} \cap B_{2}, B_{1} \cap B_{3}$, and $B_{2} \cap B_{3}$ are nonempty, then there exists $B \in \mathcal{H}$ that includes these intersections.

(iv) The Helly number of $\left(U, \lesssim_{R}\right)$ is 2 .

Proof. The equivalence (i) $\Leftrightarrow($ ii) is an immediate consequence of $[3$, Theorem 2.9] as we already noted, and the implication (ii) $\Rightarrow$ (iii) is clear by Remark 4.3 .

(iii) $\Rightarrow$ (iv): Let $R\left(a_{1}\right), R\left(a_{2}\right)$, and $R\left(a_{3}\right)$ be minimal elements in $\mathcal{N}$ such that the intersections $R\left(a_{1}\right) \cap R\left(a_{2}\right), R\left(a_{1}\right) \cap R\left(a_{3}\right)$, and $R\left(a_{2}\right) \cap R\left(a_{3}\right)$ are nonempty. Then, the elements $a_{1}, a_{2}$, and $a_{3}$ are minimal in $\left(U, \lesssim_{R}\right)$ and $\uparrow a_{1}, \uparrow a_{2}$, and $\uparrow a_{3}$ belong to $\mathcal{H}$, since $\mathcal{H}$ is an irredundant covering. We have that the intersections $\uparrow a_{1} \cap \uparrow a_{2}, \uparrow a_{1} \cap \uparrow a_{3}$, and $\uparrow a_{2} \cap \uparrow a_{3}$ are nonempty, which by assumption (iii) implies that there exists a minimal $a$ in $\left(U, \lesssim_{R}\right)$ such that $\uparrow a \in \mathcal{H}$ includes the intersections $\uparrow a_{1} \cap \uparrow a_{2}, \uparrow a_{1} \cap \uparrow a_{3}$, and $\uparrow a_{2} \cap \uparrow a_{3}$. Therefore, by Proposition 4.2, the Helly number of $\left(U, \lesssim_{R}\right)$ is two.

(iv) $\Rightarrow($ i). Assume that (iv) holds. Then, by Theorem 4.1, the blocks of the tolerance $\approx_{R}$ form an irredundant covering of $U$ and $\mathcal{B}\left(\approx_{R}\right)=\{\uparrow m \mid$ $m$ is minimal $\}$. Since by assumption, $R$ is induced by an irredundant covering $\mathcal{H}$, Theorem 3.4 implies that $R$ equals $\approx_{R}$. By Lemma 2.3, we have $\mathcal{H} \subseteq \mathcal{B}(R)=\mathcal{B}\left(\approx_{R}\right)$. Because both $\mathcal{H}$ and $\mathcal{B}\left(\approx_{R}\right)$ are irredundant coverings inducing $R$, we get $\mathcal{H}=\mathcal{B}(R)$.

Example 4.5. Let us consider the tolerance on $U=\{a, b, c, d, e, f, g\}$ given in Figure 2, The corresponding quasiordered set $\left(U, \lesssim_{R}\right)$ is bounded by minimal elements $a, c, g$. In addition, the tolerance $R$ coincides with the relation $\approx_{R}$ and thus $R$ is induced by an irredundant covering $\mathcal{H}=\{\uparrow a, \uparrow c, \uparrow g\}$. 


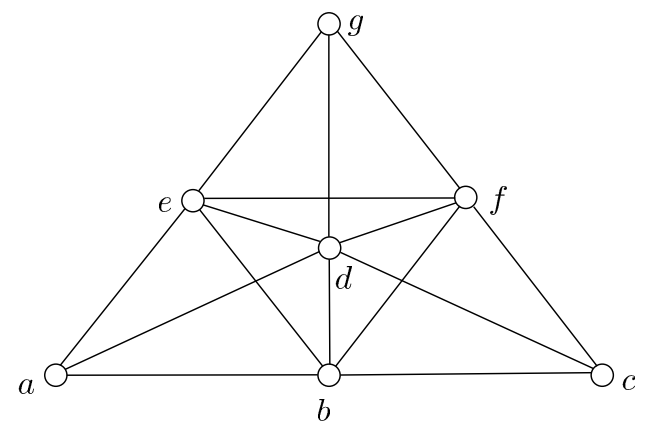

Tolerance $R$

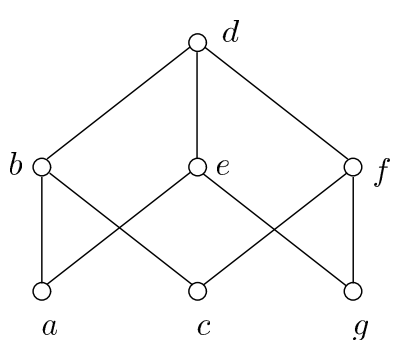

$\left(U, \lesssim_{R}\right)$

FiguRE 2.

We have that the following intersections of elements in $\mathcal{H}$ are nonempty:

$$
\uparrow a \cap \uparrow c=\{b, d\}, \uparrow a \cap \uparrow g=\{d, e\}, \text { and } \uparrow c \cap \uparrow g=\{d, f\} .
$$

Obviously, there is no $B \in \mathcal{H}$ that includes the union $\{b, d, e, f\}$ of the above intersections. By Proposition 4.4, we have $\mathcal{H} \subset \mathcal{B}(R)$. In fact, $\{b, d, e, f\}$ is the only block of $R$ not belonging to $\mathcal{H}$.

It is also interesting to notice that the quasiordered set $\left(U, \lesssim_{R}\right)$ of Figure 2 is almost isomorphic to the Boolean lattice $\mathbf{2}^{\mathbf{3}}$ except that the least element is missing. Next we consider the tolerances induced by finite distributive lattices. Let $\mathcal{L}=(L, \leq)$ be a lattice with a least element 0 . We denote the set $L \backslash\{0\}$ by $L^{+}$. We say that the Helly number of the lattice $\mathcal{L}$ is $k$, if the partially ordered set $\left(L^{+}, \leq\right)$has the Helly number $k$.

Corollary 4.6. If $\mathcal{L}=(L, \leq)$ is a finite distributive lattice, then the Helly number of $\mathcal{L}$ is 2 if and only if $\mathcal{L}$ has at most two different atoms.

Proof. Because $\mathcal{L}$ is finite, it has a least element 0, and its atoms are precisely the minimal elements of $\left(L^{+}, \leq\right)$. If their number is one or two, then condition (b) of Proposition 4.2 is trivially satisfied, and hence the Helly number of $\left(L^{+}, \leq\right)$and $\mathcal{L}$ is 2 .

Conversely, suppose that the Helly number of $\mathcal{L}$ is 2 . Let us first recall the well-known facts that for any $x, y \in L, \uparrow x \cap \uparrow y=\uparrow(x \vee y)$, and $x \geq y$ if and only if $\uparrow x \subseteq \uparrow y$.

Let $a_{1}, a_{2}, a_{3} \in L$ be three distinct atoms of $\mathcal{L}$. First notice that they are minimal elements in $\left(L^{+}, \leq\right)$and that the upsets corresponding to these atoms can not be disjoint, because each of them contains the greatest element 1 of the finite lattice $\mathcal{L}$. By applying Proposition 4.2 , we get that there exists an atom $a \in L$ such that $\uparrow\left(a_{1} \vee a_{2}\right) \subseteq \uparrow a, \uparrow\left(a_{1} \vee a_{3}\right) \subseteq \uparrow a$, and $\uparrow\left(a_{2} \vee a_{3}\right) \subseteq \uparrow a$. Since $L$ is distributive and $a_{1}, a_{2}, a_{3}$ are atoms, this implies $a \leq\left(a_{1} \vee a_{2}\right) \wedge\left(a_{1} \vee a_{3}\right) \wedge\left(a_{2} \vee a_{3}\right)=\left(a_{1} \wedge a_{2}\right) \vee\left(a_{1} \wedge a_{3}\right) \vee\left(a_{2} \wedge a_{3}\right)=0$, a contradiction.

We may now conclude that each finite distributive lattice $\mathcal{L}=(L, \leq)$ induces a tolerance $(\geq 0 \leq)$ on $L^{+}$. Let us denote this tolerance by $\bowtie$. Because $L^{+}$is finite, it is trivially bounded by minimal elements. By Lemma 3.1 , 
$\mathcal{H}=\{\uparrow m \mid m$ is minimal $\}$ is an irredundant covering of $L^{+}$, and the tolerance induced by $\mathcal{H}$ is $\bowtie$. By Corollary 4.6 , the Helly number of $\mathcal{L}$ is 2 if and only if $\mathcal{L}$ has at most two different atoms. Finally, we obtain that $\mathcal{B}(\bowtie)=\mathcal{H}$ if and only if $\mathcal{L}$ has at most two different atoms by Proposition 4.4 .

\section{ACKNOWLEDGEMENTS}

We thank the anonymous referees for their valuable remarks on our manuscript.

\section{REFERENCES}

[1] J. Alt and N. Schofield, Clique analysis of a tolerance relation, The Journal of Mathematical Sociology 6 (1978), 155-162.

[2] W. Bartol, J. Miró, K. Pióro, and F. Rosselló, On the coverings by tolerance classes, Information Sciences 166 (2004), 193-211.

[3] I. Chajda, Algebraic Theory of Tolerance Relations, Monograph series of Palacký University, 1991.

[4] I. Chajda, J. Niederle, and B. Zelinka, On existence conditions for compatible tolerances, Czechoslovak Mathematical Journal 26 (1976), 304-311.

[5] G. Czédli, Factor lattices by tolerances, Acta Scientiarum Mathematicarum (Szeged) 44 (1982), 35-42.

[6] G. Czédli and L. Klukovits, A note on tolerances of idempotent algebras, Glasnik Matematički 18 (1983), 35-38.

[7] B. Ganter and R. Wille, Formal concept analysis: Mathematical foundations, Springer, Berlin/Heidelberg, 1999.

[8] G. Grätzer and G. H. Wenzel, Tolerances, covering systems, and the axiom of choice, Archivum Mathematicum 25 (1989), 27-34.

[9] F. Harary and I. C. Ross, A procedure for clique detection using the group matrix, Sociometry 20 (1957), 205-216.

[10] J. Järvinen, Lattice theory for rough sets, Transactions on Rough Sets VI (2007), 400-498.

[11] J. Järvinen and S. Radeleczki, Rough sets determined by tolerances, International Journal of Approximate Reasoning 55 (2014), 1419-1438.

[12] J. Nieminen, Screens and rounding mappings, Zeitschrift für Angewandte Mathematik und Mechanik 58 (1978), 519-520.

[13] _ Blocks, error algebras and flou sets, Glasnik Matematički 14 (1979), 381-385.

[14] J. Pogonowski, Tolerance spaces with applications to linguistics, University Press, Institute of Linguistics, Adam Mickiewicz University, Poznań, 1981.

[15] Ju. A. Schreider, Equality, Resemblance, and Order, Mir Publishers, Moskow, 1975.

[16] Y. Yao and B. Yao, Covering based rough set approximations, Information Sciences 200 (2012), 91-107.

[17] E. C. Zeeman, The topology of the brain and visual perception, Topology of 3Manifolds (M. K. Fort, ed.), Prentice-Hall, Englewood Cliffs, NJ, 1962.

[18] B. Zelinka, Tolerance graphs, Commentationes Mathematicae Universitatis Carolinae 9 (1968), 121-131.

[19] — A remark on systems of maximal cliques of a graph, Czechoslovak Mathematical Journal 27 (1977), 617-618.

J. JärVinen, Sirkankuja 1, 20810 Turku, Finland

E-mail address: Jouni.Kalervo.Jarvinen@gmail.com

$U R L:$ http://sites.google.com/site/jounikalervojarvinen/

S. Radeleczki, Institute of Mathematics, University of Miskolc, 3515 MiskolC-Egyetemváros, Hungary

E-mail address: matradi@uni-miskolc.hu

$U R L:$ http://www.uni-miskolc.hu/ matradi/ 